\title{
Grading and surgical planning for intracranial arteriovenous malformations
}

\author{
Howard A. RiIna, M.D., AND Y. Pierre Gobin, M.D. \\ Departments of Neurological Surgery and Radiology, Weill/Cornell Medical College, New York, \\ New York
}

\begin{abstract}
The treatment of arteriovenous malformations (AVMs) has evolved over the last 40 years. These complex vascular lesions remain among the most difficult lesions to treat. Successful treatment of AVMs of the brain includes extensive preoperative planning, multimodality treatment options, and modern postoperative surgical care. The advent of new technologies, including interventional neuroradiology and radiosurgery, has expanded the range of malformations that can be treated effectively and has had a significant impact on those individuals who manifest this disease process. The purpose of this paper is to describe the current grading technique used by the authors and to explore the preoperative treatment and planning that leads to successful surgical obliteration of these lesions. Some description of preoperative interventions, including radiosurgery and interventional procedures will be mentioned; however, only in the context of how they impact on the surgical treatment of these lesions. In other articles in this edition of Neurosurgical Focus interventional procedures and radiosurgery as treatment adjuncts and as primary therapies will be discussed in greater detail.
\end{abstract}

\section{KEY WORDS • arteriovenous malformation - microneurosurgery •} interventional neuroradiology $\bullet$ embolization $\bullet$ radiosurgery

Arteriovenous malformations of the brain remain among the most complex and difficult lesions that cerebrovascular neurosurgeons must treat. Their successful obliteration requires intense preoperative planning, meticulous adherence to microneurosurgical technique, and state-of-the-art postoperative care. Preoperative planning, including therapies that can make difficult lesions more amenable to surgery (interventional neuroradiology and radiosurgery) are all part of the treatment algorithm for these lesions. Currently, the majority of neurosurgeons, interventional neurosurgeons, and interventional radiologists who treat AVMs of the brain use the Spetzler-Martin grading system. ${ }^{3}$ The Spetzler-Martin grading system is used to grade AVMs according to size, location, and venous drainage. This grading system has, for over a decade, served as a mode of communication between neurosurgeons and other subspecialties involved in the treatment of these complex lesions. The initial step in grading an AVM has implications for surgical resectability and patient outcome. It is possible, by using adjuvant therapies including embolization and radiosurgery, to convert higher-grade

Abbreviations used in this paper: $\mathrm{AVM}=$ arteriovenous malformation; $\mathrm{CSF}=$ cerebrospinal fluid; $\mathrm{CT}=$ computerized tomography; EEG = electroencephalography; MR = magnetic resonance. lesions to lower-grade lesions that are more amenable to surgical obliteration. Once the decision has been made to resect an AVM surgically, the formal planning of the procedure should be performed. Prior to this step, all available imaging studies that could contribute to a successful surgical procedure should be obtained. These include MR imaging, MR angiography, CT scanning, CT angiography, and four-vessel angiography. Imaging data can then be used as a guide for resection. Once resection has begun, meticulous attention to the microneurosurgical procedure is imperative to a successful outcome. The goal is to resect the AVM without interfering with normal vascular supply and normal brain parenchyma. Once the patient has undergone resection of the AVM, postoperative care is as important in achieving a successful outcome. This monograph will describe the grading of cerebral AVMs, the successful planning and execution of resection of cerebral AVMs, and the postoperative care of these patients once they have undergone surgery.

\section{GRADING SYSTEM}

The Spetzler-Martin grading system was described more than 15 years ago and has become one of the most widely accepted and useful grading systems for the com- 


\section{H. A. Riina and Y. P. Gobin}

munication of information regarding intracerebral AVMs. In the grading system, AVMs receive a grade of I through $\mathrm{V}$, which corresponds to a point scale depending on the size of the lesion, the eloquence of adjacent brain tissue, and the pattern of venous drainage. The importance of the grading system is that it has significant implication for surgical outcome. Higher-grade lesions, being more difficult to resect, have higher degrees of patient morbidity and mortality associated with their resection. As mentioned earlier, it is possible, by using some of the newer methods associated with endovascular therapy and radiosurgery, to decrease the size of the lesions, thus reducing their grade and making their resection possible with lower morbidity.

\section{Preoperative Planning}

The preoperative planning stage begins with the assessment of the patient. The neurological evaluation of the patient is important in determining the preoperative neurological status. Any neurological deficits must be highlighted in detail, as all the therapies that we as clinicians use are not without risk. Once the neurological examination and evaluation have been performed, we can begin the process of acquisition of anatomical data regarding the AVM.

The technological advancement in imaging capabilities that has occurred over the last 30 years has greatly enhanced our ability to characterize these lesions and more adequately understand their natural history. As an initial imaging examination, many patients will undergo an unenhanced CT scan. This plain CT scan of the brain will often reveal changes that have occurred secondary to the AVM. These can include the presence of large drainage veins or varices, areas of calcification related to previous hemorrhage, and of course any acute hemorrhage and edema in the brain parenchyma around the lesion. A CT scanning evaluation of the brain is usually followed by an MR study and often MR angiography is performed in the same sitting. Magnetic resonance imaging data produces more precise size and anatomical localization information on the AVM nidus; however, oftentimes it is difficult to delineate the true nidus of the AVM from the draining venous structures based on MR imaging and MR angiography alone. The preliminary grade can be assigned based on MR imaging and MR angiography data; however, this may have to be modified once four-vessel angiography is performed. Magnetic resonance imaging of the brain is also extremely useful in the postoperative period as well as during the follow-up period.

Because these are vascular lesions, the most important preoperative study that can be obtained is four-vessel angiography. Four-vessel digital subtraction angiography remains the gold standard in delineating the vascular anatomy. The precise size and location of an AVM once correlated with MR imaging data leads to appropriate grading and preoperative planning. More importantly, the size, location, and feeding vessels to the nidus of the AVM are clearly delineated as are all venous structures related to the malformation. The normal vascular anatomy of the surrounding brain is also demonstrated by these studies. One of the tenets of microneurosurgical resection of these lesions involves preservation of the normal vascular anatomy as this has implications for the normal tissue in adjacent areas. Four-vessel diagnostic angiography can also be performed at the time of endovascular intervention as this reduces risk by decreasing the number of procedures that a patient must undergo. In some instances, CT angiography may provide additional information regarding bone anatomy and its relation to a particular malformation. We have found CT angiography more useful in surgical planning for intracranial aneurysms than in the treatment of AVMs of the brain.

\section{EMBOLIZATION TECHNIQUES}

The development and improvement in endovascular techniques has greatly enhanced our ability to resect AVMs of the brain. This can often be performed at the time of a diagnostic angiogram, but more often is performed as a second or staged procedure. Improvement in microguidewire and microcatheter technology has increased our ability to cannulate the vessels contributing to the nidus of the AVM. Additionally, improvements in embolization therapies, such as electrically detachable coils, liquid coils, pushable coils, and liquid embolic agents, have enhanced our ability to treat these lesions from an endovascular approach. The goal of embolization is to decrease the size of the nidus, decrease blood flow to the AVM, and facilitate its removal. Embolization may also have a role in the treatment of higher-grade AVMs in patients who are deteriorating neurologically. It is often possible with embolization to reduce the preoperative grade of an AVM so that the patient morbidity and mortality associated with the surgical removal of the lesion is lessened as well. Embolization is not without risk. It should only be performed when there is a clear indication for improving the natural history of the AVM in question. This is achieved by either improving patient symptoms, decreasing the grade of the lesion so it is amenable to other therapies or by obliterating the lesion entirely.

Some Grade I and II AVMs may be entirely obliterated with embolization just as some may be completely removed surgically without preoperative embolization. At our institution, a multidisciplinary approach is taken toward the treatment of AVMs. Cerebrovascular surgeons, interventional neurosurgeons, and interventional neuroradiologists all discuss the management of each case and recommend a treatment algorithm based on the findings of the group. We find that the pooling of this expertise is beneficial and improves patient outcome.

\section{RADIOSURGICAL TREATMENT}

Stereotactic radiosurgery also has a well-documented role in the treatment of cerebral AVMs. Like endovascular therapy, radiosurgery can be used to obliterate small lowgrade lesions and to decrease the size of higher-grade lesions so that they are then amenable to either endovascular or surgical intervention. It is not unusual to have a single AVM that has been treated using all three modalities. Radiosurgery does require a significant period of time, approximately 2 years, before the effects of the focused radiation are seen. ${ }^{4,5}$ During this time, the patient is still at risk and subject to the natural history of the AVM as if it were untreated. 


\section{MICRONEUROSURGICAL RESECTION}

Resection of AVMs requires adherence to the basic tenets of microneurosurgical technique. These include meticulous technique, preservation of normal vascular anatomy, minimization of brain retraction, and cerebral protection when necessary.

\section{Operative Approach}

Once the decision is made for the patient to undergo resection of the AVM, the correct approach must be chosen. This is based solely on anatomical considerations. We often favor a wide craniotomy and foster any maneuvers that increase exposure. It is assumed that a wide dural opening follows a wide craniotomy. Once the craniotomy is performed, the operating microscope is brought into the field, and the microneurosurgical resection of the lesion is performed. We often have retractors available to aid in exposure; however, we favor CSF drainage to help minimize brain retraction. Drainage of CSF can be accomplished using either a ventriculostomy in patients who have presented with an intraventricular hemorrhage or hydrocephalus and straight lumbar drainage in those who do not have a ventricular drain. We will often open the lumbar drain prior to the dural opening. With adequate brain relaxation, it is often not necessary to use retractors and thus the surrounding brain is often protected.

Cerebral protection can also be of use in the resection of these complex lesions. ${ }^{1,2}$ When the lesion involves eloquent cortex and often at times when it does not, we will use burst suppression therapy with either barbiturates or Diprivan. Both of these medications can be titrated to burst suppression on EEG monitoring obtained continuously throughout the procedure. The actual resection of the lesion requires a thorough understanding of the arterial feeding vessels and venous drainage. An often-stated goal is to remove sequentially the arterial feeding vessels that supply the nidus and then to take the main draining vein last. If the venous drainage is interrupted prior to substantial reduction of the arterial flow, the nidus may rupture and significant bleeding may be encountered. We prefer to work our way around the nidus of the lesion, gradually removing arterial feeding vessels and decreasing the size of the nidus. Large feeding pedicles often have to be clipped with microneurosurgical AVM clips but frequently may just be held with bipolar forceps. It is often necessary to continually reference the angiogram so that one gets a three-dimensional understanding of the microscopic view. This is particularly useful after the nidus has been removed so that any residual malformation can be identified. Once the major arterial feeding vessels have been disconnected from the nidus and the nidus has been sequentially devascularized, the venous drainage can then be disconnected. The large draining venous pedicles are the last vessels that are disconnected from the AVM. Once the nidus has been removed, the resection bed is inspected for any residual nidus or abnormal-appearing vessels. We then like to ask the anesthesiologist to elevate the blood pressure so that we can test the resection bed and look for potentially hazardous areas. Any suspect areas that begin bleeding can be explored and any abnormal vasculature in the area can be removed. In some of our more difficult cases, intraoperative angiography is performed. This is useful in identifying areas of residual malformation at the time of surgery. The images obtained from intraoperative angiography are not as good as those obtained in the angiography suite. If there are remaining questions, we may take the patient right from the operating room to the angiography suite to undergo a standard study. The patient can then be brought back to the operating room if any questions remain.

\section{Anatomical Considerations}

Supratentorial lobar AVMs should be approached through a wide craniotomy so that the surgeon has plenty of operating room. Low-grade lesions, often in the frontal lobe, can be resected with good results even though there must be some manipulation of the surrounding parenchyma. As the grade of the lesion increases (size and location adjacent to eloquent cortex) care must be taken not to damage the surrounding parenchyma. In particular, patients with lesions involving the motor cortex or speech cortex should undergo functional MR imaging prior to surgery, and the surgeon may wish to consider performing the operation while the patient is awake, with motor mapping or phase reversal. We do not routinely recommend the treatment of Grade IV or V AVMs unless the patient is deteriorating neurologically or if the patient has had multiple hemorrhagic events. The staged treatment of giant lesions, Grades IV and V, by using endovascular therapies and radiosurgery as mentioned earlier, may decrease the grade and make previously unresectable lesions amenable to surgery.

A particular mention must be made of AVMs occurring within the sylvian fissure. These malformations are often within the subarachnoid space, and their resection can be performed without risking the surrounding parenchyma. The surgical treatment of these lesions, however, requires meticulous understanding of the normal vasculature in the region so that normal vessels are not interrupted. We have found that intraoperative angiography is of particular use in the treatment of these lesions.

In the treatment of infratentorial AVMs, one must distinguish between those occurring within the cerebellar hemispheres and those involving the brainstem. Arteriovenous malformations involving the brainstem are difficult because of the eloquence of the territory involved, but their resection is possible. In obliterating vessels that supply the nidus of these lesions, one must be sure that they go directly into the AVM and are not supplying normal tissue. These vessels can often be followed directly into the nidus of the AVM. Monitoring brainstem auditory evoked responses is a useful method in the treatment of these lesions. As long as the surgeon operates within the cavity of the AVM, the surrounding parenchyma should not be at risk. One should try to obtain the greatest exposure possible by using the transpetrosal, far-lateral, and occipital approaches, as necessary. Lesions involving the hemispheres of the cerebellum are more amenable to resection than lesions of the brainstem. One can work around the nidus of a malformation within the parenchyma of the cerebellum so that the nidus can be circumscribed. When operating in the posterior fossa, it is often useful to open up the cisterna magna to allow adequate CSF drainage re- 
sulting in significant brain relaxation. Preoperative embolization of any AVM may help in the identification of arterial feeding vessels to the AVM, as the glue cast seen within the feeding vessels can be identified. At times, it may be difficult to identify arterialized venous structures. Although prior to sacrificing any AVM, large vessels should be temporarily held either within the tips of the bipolar forceps or with a small clip to see if there are any significant changes in the nidus prior to sacrifice. One takes a sequential approach and works around the perimeter of the nidus of a lesion. The sequential circumferential obliteration of the lesion can be achieved. The use of skull base approaches has helped in the treatment of some lesions, particularly those involving the brainstem, in that they help provide increased working room for the surgeon and minimize the need for brain retraction. The goal of any AVM operation is to remove the entire nidus. If the nidus is not removed, the risk of hemorrhage remains.

\section{Intraoperative Monitoring}

Intraoperative monitoring has become a useful method in the surgical management of AVMs. Brainstem auditory evoked response, cranial nerve, and EEG monitoring at all times can be useful during the resection period. Cortical mapping of lesions that are near or in the motor strip is also particularly useful at the time of surgery. Additionally, EEG can be useful to the anesthesiologist when burst suppression is induced in patients for cerebral protection.

\section{POSTOPERATIVE CARE}

The immediate postoperative period in the management of surgical patients is of supreme importance. We like to maintain normotension in the patients during the postoperative period. The patient should undergo frequent neurological evaluation in a neurosurgical intensive care unit setting. Any changes in the neurological examination should be followed by prompt CT scan evaluation. Postsurgery, the patient is at risk for seizures, rebleeding, and swelling secondary to hyperemia. When a patient rebleeds, one must consider either incomplete resection of the nidus or perfusion pressure-type breakthrough bleeding. Rebleeding should prompt repeated angiography and reoperation. All patients undergoing successful resection of their AVM should undergo postoperative angiography to document that their malformation has been entirely resected. The patient with an incompletely resected or staged em- bolization or radiosurgery procedure in addition should undergo MR imaging.

\section{CONCLUSIONS}

The successful treatment of AVMs requires a multidisciplinary approach involving cerebrovascular neurosurgeons, endovascular neurosurgeons, interventional radiologists, and neurointensivists. Critical to the successful outcome of the surgery of these lesions, intense preoperative planning by using all modes of neuroimaging available and the information that they provide is required. Preoperative superselective embolization as well as radiosurgery play significant roles in the staged approach to the management of these lesions and in making high-grade lesions lower in grade and more suitable to resection. Meticulous attention to the details of microneurosurgical technique must be adhered to once surgery has been performed. These include meticulous dissection, preservation of all normal vascular structures, minimized retraction and injury to surrounding brain, wide exposure, adequate brain relaxation, and cerebral protection. Intraoperative monitoring is useful and may help to steer the surgeon from eloquent regions. All these factors contribute to the successful outcome in patients with AVMs of the brain.

\section{References}

1. Selman WR, Spetzler RF, Ruski RA, et al: Barbiturate coma in focal cerebral ischemia. Relationship of protection to timing of therapy. J Neurosurg 56:685-690, 1982

2. Spetzler RF, Hadley MN: Protection against cerebral ischemia: the role of barbiturates. Cerebrovasc Brain Metab Rev 1: 212-229, 1989

3. Spetzler RF, Martin NA: A proposed grading system for arteriovenous malformations. J Neurosurg 65:476-483, 1986

4. Steiner L, Lindquist C, Adler JR, et al: Clinical outcome of radiosurgery for arteriovenous malformations. J Neurosurg 77: $1-8,1992$

5. Steiner L, Lindquist C, Cail W, et al: Microsurgery and radiosurgery in brain arteriovenous malformations. J Neurosurg 79: 647-652, 1993

Manuscript received October 20, 2001.

Accepted in final form October 25, 2001.

Address reprint requests to: Howard A. Riina, M.D., Departments of Neurological Surgery and Radiology, Weill/Cornell Medical College, 525 East 68th Street, Box 99, New York, New York 10021.email: har9005@med.cornell.edu. 